\title{
Electrical Load Forecasting using ARIMA, Prophet and LSTM Networks
}

\author{
Durga Prasad Ananthu ${ }^{\mathbf{1}}$ and Prof. Neelashetty $\mathbf{K}^{\mathbf{2}}$ \\ ${ }^{1}$ Research Scholar, VTU-RRC, Belagavi, India \\ ${ }^{2}$ Professor, DoEE, GND, Bidar, India \\ *Correspondence: Durga Prasad Ananthu; Email: adp.ananthu@ gmail.com
}

\begin{abstract}
Forecasting electrical load plays a vital role in power system planning. However, it is quite difficult to forecast electrical load, as the load on the system varies continuously concerning time and seasons. In this paper, we are proposing an advanced artificial neural network model to forecast short-term electrical load. The proposed method tested on historical data collected from Karnataka power corporation, India, and test results compared with other data-driven models viz. ARIMA, RNN, LSTM, and Prophet. The accuracy and RMSE values were calculated and observed that the proposed model was superior in a day and weekly ahead electrical load forecasting.
\end{abstract}

Keywords: Load forecasting, ARIMA, RNN, LSTM (Long Short-Term Memory), Prophet, Artificial Neural Network (ANN), Power System Planning.

\begin{tabular}{|c|c|}
\hline \multicolumn{2}{|l|}{ ARTICLE INFORMATION } \\
\hline \multicolumn{2}{|c|}{$\begin{array}{l}\text { Author(s): Durga Prasad Ananthu, Prof. Neelashetty K } \\
\text { Received: Dec 21, 2021; Accepted: Jan 25, 2022; Published: Feb 05, 2022; }\end{array}$} \\
\hline $\begin{array}{l}\text { e-ISSN: 2347-470X; } \\
\text { Paper Id: IJEER120121; }\end{array}$ & crossef member \\
\hline $\begin{array}{l}\text { Citation: doi.org/10.37391/IJEER.090404 } \\
\text { Webpage-link: }\end{array}$ & $\begin{array}{l}\text { CROSSRER.ORG } \\
\text { THECTATION LNKING BACKBONE } \\
\end{array}$ \\
\hline
\end{tabular}

\section{INTRODUCTION}

The demand of electrical energy is increasing day by day, supplying electricity to all the stakeholders is challenging in countries like India, as it is a fast developing nation. The load on the grid varies with respect to time, to meet these demand utilities need to estimate the consumption demand priority. This can be possible by using AI/ML techniques to estimate the load demand accurately. The advantages of load forecasting is that it helps in reducing outages, losses, overall operating cost and improves the stability, reliability, power quality and also helps in power system planning. It plays a major role in implementing demand side management.

[1] In this paper they proposed a method to forecast the load using a correlation analysis and i2 input selection method. Euclidian distance is used for BNN (Bayesian neural network) training. Forecast accuracy, and error were measured. [2] Proposed a GABICS (Genetic algorithm binary improved cuckoo search) with extreme learning machine techniques to forecast the Short term load by using date framework with feature selection method. [3] Presented a SWEMD (Sliding window empirical mode decomposition) model for building load forecasting this method used for small sites. [4] Used support vector regression analysis for day ahead LF. [5] Presented feature engineering and modified firefly optimization algorithm with SVR model for STLF (Short-term load forecasting). Ref. [6] presented a comparison study between ARIMA and LSTM models to forecast daily load for smart homes using STDAN algorithm. [7] Proposed multistep forecasting using autoregressive and integrated MA and RNNLSTM methods for nonlinear loads.

In this paper we have collected Karnataka State load consumption data for various seasons; the data set contains 360 samples to forecast the load in a day and week ahead of time. The data is available at "http://218.248.45.137:8282/LoadCurveUpload/lcdownloadvie w.asp".

The flow of work is as follows, section 1 gives the literature survey/Background, section 2 discusses the implementation of load forecasting using LSTM networks section 3 deals with the other forecasting models like ARIMA, SARIMA, RNN and Prophet Section 4 discusses results and conclusion.

\section{BACKGROUND}

Xin Liu et al. [8] elaborated various data driven models to forecast short term electrical load in this they used GFW based feature selection to determine the correct predictions and these results are compared with the DNN model using shallow neural networks. Elvisa and Marijana [9] proposed different machine learning algorithms to forecast electrical load and implemented additive regression and ANN analysis on STL forecasting for summer and winter seasons. Wan $\mathrm{He}$ [10] presented DNN technique to predict one day ahead loads and developed CNN models to extract features in historical load. Ammar $\mathrm{O}$ et al. [11] in this paper used MC(Multicolumn) radial basis function NN for STLF. They implemented $\mathrm{k}-\mathrm{d}$ tree algorithm using modified error correction method to form MCRN, this technique improves the speed of convergence and improves the generalization over other methods. Stefan Hosein et al. [12] in this they applied DNN(Deep neural network) and other machine learning techniques on SMART Meter data to STLF in a power grid and also discussed dynamic pricing to 
reduce peak load. Calculated daily MAPE and compared with other models. Sajjad Khan et al. [13] presented "empirical mode decomposition based extreme learning machine" technique for daily, weekly and monthly building load forecasting and results compared with CNN model. Hossein Javedani Sadaei et al.[14] presented a method using fuzzy time series and convolutional neural networks to forecast STL Dagdougui H.et al.[15] Proposed NN model using Bayesian and Levenberg learning algorithms to forecast short-term load in buildings.

From the related research it is observed that the previous presented models are having high MAP error, mean squared error and RMSE. So it is necessary to develop a model with low MAPE.MSE and RMSE to STLF with high accuracy. For that we developed various deep learning methods like LSTM, Prophet, ARMA, SARIMA and RNN models to forecast STL and compared the test results with each other. We have collected the historical data from the Karnataka state power corporation Ltd., India.

\section{IMPLEMENATION OF LSTM NETWORKS}

LSTM networks outperforms in neural network family compare to other neural networks, it has a memory cell to store the data. This feature helps in analysing the large time series data. It has input gate, forget gate, output gate and a cell candidate gate. The function of output gate is to read the information from the memory cell, input gate feeds the information to cell and forget gate will decide which information to forget and retain.At the beginning of each time $t$, the unit first calculates the activation function $\left\{i_{\mathrm{t}}, f_{\mathrm{t}}\right\}$ of the input gate and the forget gate, and updates the memory cell from $c_{\mathrm{t}-1}$ to $c t$ [16].

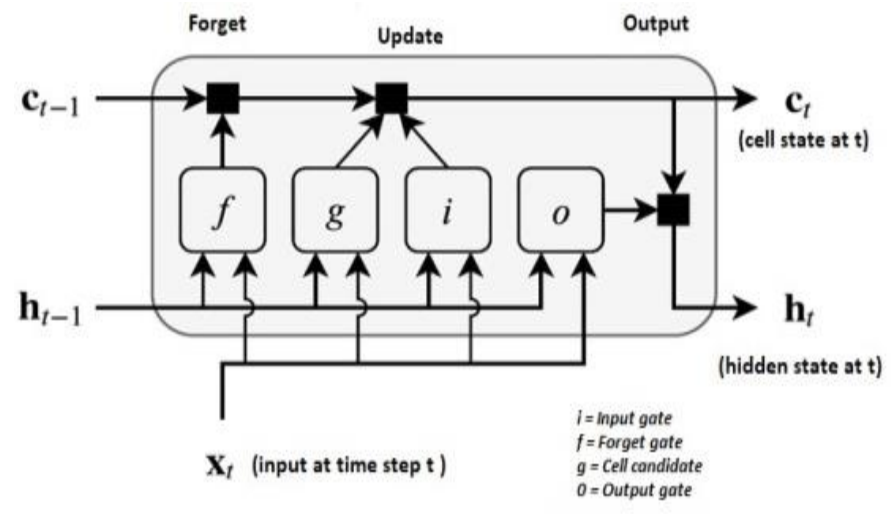

Figure 1: LSTM RNN

$\mathrm{i}_{\mathrm{t}}=\sigma\left(\mathrm{W}_{\mathrm{i}} \mathrm{x}_{\mathrm{t}}+\mathrm{U}_{\mathrm{i}} \mathrm{H}_{\mathrm{t}-1}+\mathrm{V}_{\mathrm{i}} \mathrm{C}_{\mathrm{t}-1}+\mathrm{b}_{\mathrm{i}}\right)$

$\mathrm{f}_{\mathrm{t}}=\sigma\left(\mathrm{W}_{\mathrm{f}} \mathrm{x}_{\mathrm{t}}+\mathrm{U}_{\mathrm{f}} \mathrm{H}_{\mathrm{t}-1}+\mathrm{V}_{\mathrm{f}} \mathrm{C}_{\mathrm{t}-1}+\mathrm{b}_{\mathrm{f}}\right)$

$\mathrm{C}_{\mathrm{t}}=\mathrm{f}_{\mathrm{t}} \odot \mathrm{C}_{\mathrm{t}-1}+\mathrm{i}_{\mathrm{t}} \odot \tanh \left(\mathrm{W}_{\mathrm{c}} \mathrm{x}_{\mathrm{c}}+\mathrm{U}_{\mathrm{c}} \mathrm{H}_{\mathrm{t}-1}+\mathrm{b}_{\mathrm{c}}\right)$

$O_{t}=\sigma\left(W_{o} x_{t}+U_{o} H_{t-1}+V_{o} C_{t-1}+b_{o}\right)$

$\mathrm{H}_{\mathrm{t}}=\mathrm{O}_{\mathrm{t}} \odot \operatorname{tanhC}_{\mathrm{t}}$
The algorithm for LSTM network given Fig2 taken from "https://in.mathworks.com/help/deeplearning/ug/time-seriesforecasting-using-deeplearning.html?s_tid=srchtitle_time $\% 20$ series $\% 20$ forecasting_1 "

\section{Load sequence data}

2. Standardize the training data to have zero mean and unit variance. 3. Prepare Predictors and Responses (XTrain,
YTrain)

4. Create an LSTM regression network

5. Train the LSTM network with the specified training options by using trainNetwork.

6. Forecast Future Time Steps (use the predictAndUpdateState function to predict time steps one at a time and update the network state at each prediction)

Standardize the test data and Unstandardize the predictions

7. Update Network State with Observed Values using resetState

\section{Calculate the RMSE}

Figure 2: LSTM networks process flow

\subsection{LSTM Results}

The Load the historical data and process it through LSTM networks, fig 3a) shows the data set info and 3b) depicts the training progress, first $90 \%$ of the data is used to train the network and remaining10\% data used for test the data. Prepare the predictors and forecast into future time steps and calculate the accuracy and RMSE value of the trained model and also estimate the future load requirement. Observed RMSE value before updating the model is $688.123 \mathrm{MW}$ and after updating the training model its value is $68.4856 \mathrm{MW}$ as shown in fig 4. The deviation in the prediction is just only a $3.2 \%$. So this method helps the utilities to plan their generation according to the load requirement and in terms increases the power quality and reliability of the distribution system.

\begin{tabular}{|c|c|}
\hline \multicolumn{2}{|l|}{ df.head() } \\
\hline & Load(in MW) \\
\hline \multicolumn{2}{|l|}{ Date_Time } \\
\hline 2021-06-15 00:00:00 & 5756.56 \\
\hline 2021-06-15 01:00:00 & 5996.09 \\
\hline 2021-06-15 02:00:00 & 5604.09 \\
\hline 2021-06-15 03:00:00 & 5730.09 \\
\hline 2021-06-15 04:00:00 & 5708.09 \\
\hline
\end{tabular}




\section{df.info()}

〈Class 'pandas.core. frame.DataFrame'〉

DatetimeIndex: 384 entries, 2021-06-15 $00: 00: 00$ to 2021-06-30 23:00:00

Freq: $H$

Data columns (total 1 columns):

Load(in MW) 384 non-null float64

dtypes: float64(1)

memory usage: $6.0 \mathrm{~KB}$

Figure 3a: KS load data set

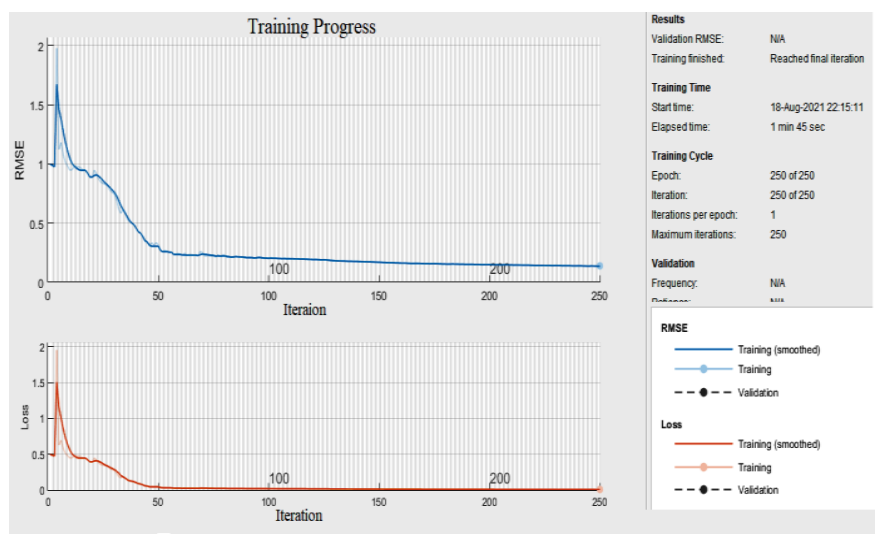

Figure 3b: Traning progess
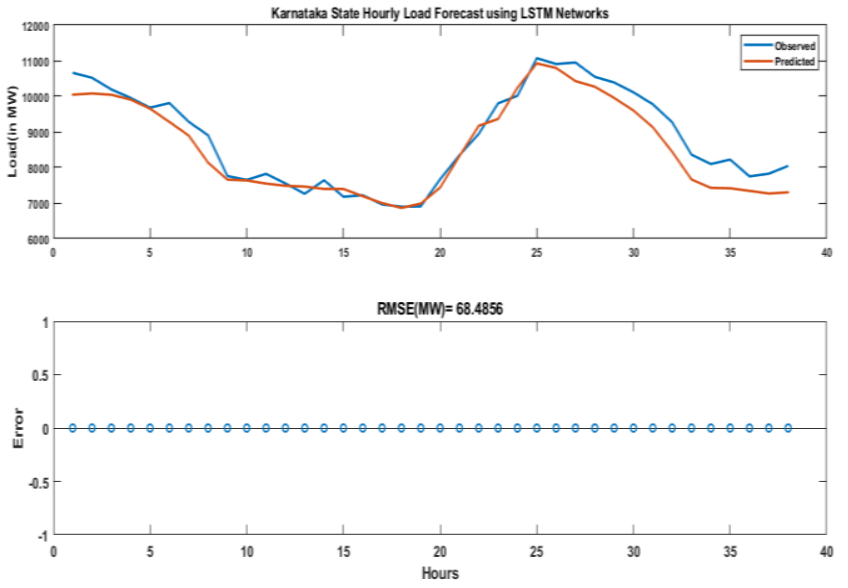

Figure 4: Hourly load forecast

\subsection{Training Parameters}

Accuracy, Sensitivity, Specificity and Precision of the trained model can be obtained from the confusion matrix shown in Fig.5. Definitions of these parameters given in the reference [17], where, Total no of samples $\mathrm{N}=1446$, truly positive sample tp $=39$, truly negative sample $t n=1396$, false positive $\mathrm{fp}=10$, false negative $\mathrm{fn}=0$, positive sample $\mathrm{p}=39$, negative sample $n=1406$.

Accuracy $=\frac{t p+t n}{N}=99.24 \%$, Sensitivity $=\frac{t p}{p}=100 \%$,

Specificity $=\frac{t n}{n}=99.29 \%$, Precision $=\frac{t p}{t p+f p}=80 \%$

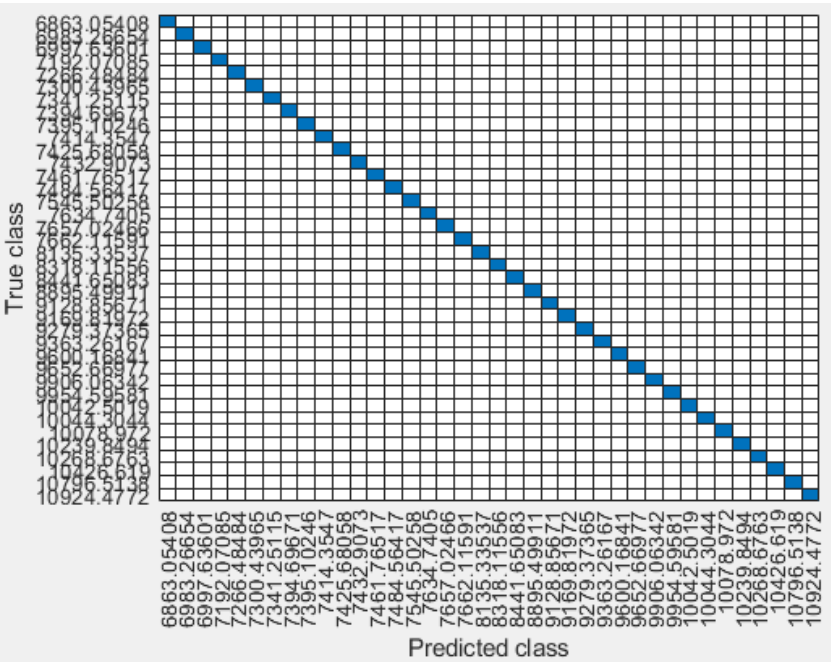

Figure 5: Confusion matrix predicted values

\section{RESULTS OF ARIMA, SARIMA, RNN AND PROPHET}

(a) ARMA Model: it has auto regressive and moving average components with an order $\mathrm{p}, \mathrm{q}$.

$X_{t}=c+\varepsilon_{t}+\sum_{i=1}^{p} \varphi_{i} X_{t-i}+\sum_{i=1}^{q} \theta_{i} \varepsilon_{t-i}$

For finding suitable p, q value we use AIC (Akaike Information criterion) or BIC (Bayesian Information criterion) criterion. The AIC and BIC values are given in Figure 6.

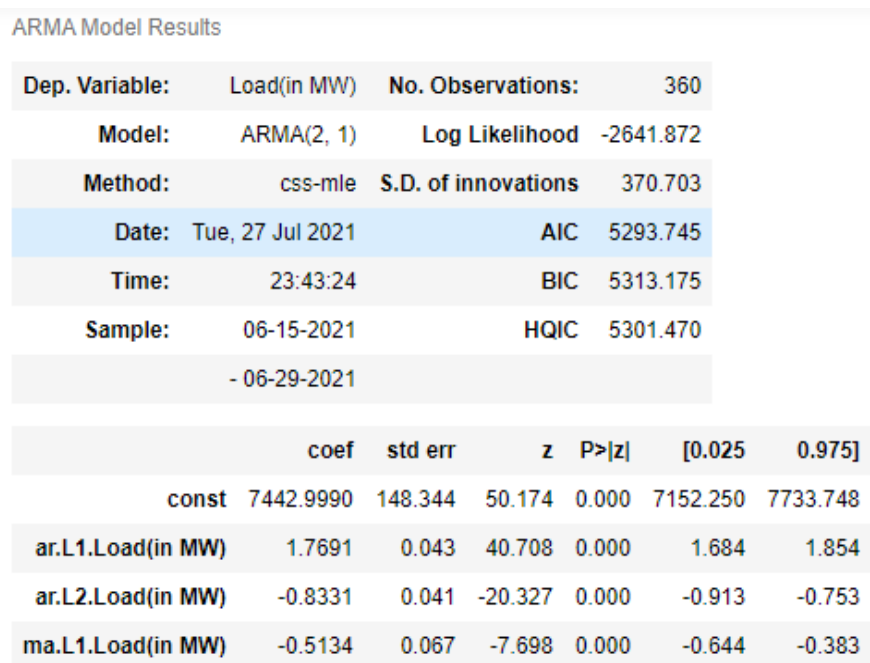

Roots

Real Imaginary Modulus Frequency

$\begin{array}{lllll}\text { AR.1 } & 1.0618 & -0.2702 \mathrm{j} & 1.0956 & -0.0397\end{array}$

$\begin{array}{lllll}\text { AR.2 } & 1.0618 \quad+0.2702 j & 1.0956 & 0.0397\end{array}$

$\begin{array}{lllll}\text { MA.1 } 1.9477 \quad+0.0000 j & 1.9477 & 0.0000\end{array}$ 


\section{ARIMA Model Results}

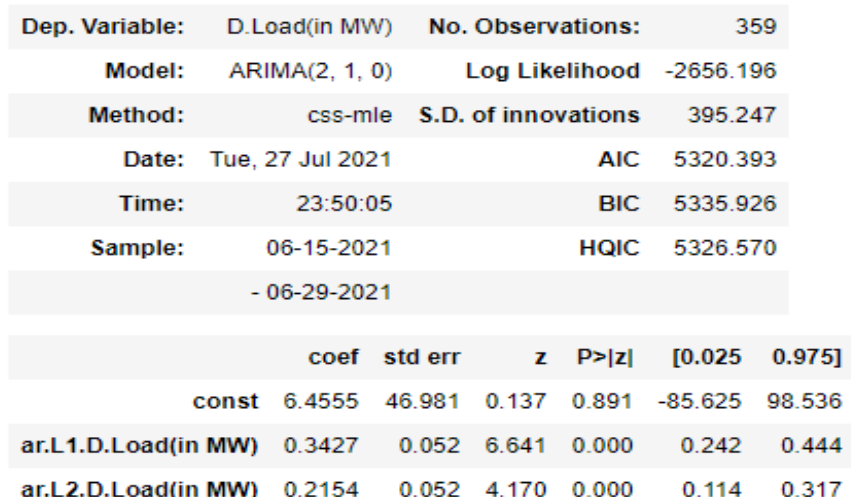

Roots

$\begin{array}{rrrrr} & \text { Real } & \text { Imaginary } & \text { Modulus } & \text { Frequency } \\ \text { AR.1 } & 1.5013 & +0.0000 \mathrm{j} & 1.5013 & 0.0000 \\ \text { AR.2 } & -3.0924 & +0.0000 \mathrm{j} & 3.0924 & 0.5000\end{array}$

Figure 6: ARMA and ARIMA Model Results

(b) ARIMA Model: it has non-seasonal and seasonal components represented by ARIMA (p,d,q) and $\operatorname{ARIMA}(\mathrm{p}, \mathrm{d}, \mathrm{q})(\mathrm{P}, \mathrm{D}, \mathrm{Q})_{\mathrm{m}}$ where $\mathrm{P}$ represents the order of AR model, $\mathrm{D}$ represents degree of differencing and $\mathrm{Q}$ indicates the order of MA model, $m$ refers the number of periods in each season [18]

$\left(1-\sum_{i=1}^{p} \varphi_{i} L^{i}\right)\left((1-L)^{d}\right) X t=\delta+\left(1+\sum_{i=1}^{q} \theta_{i} L^{i}\right) \varepsilon_{t}$

Where L is the lag operator, $\boldsymbol{\varphi}_{\boldsymbol{i}}$ is the AR parameter, $\boldsymbol{\theta}_{\boldsymbol{i}}$ is the MA parameter and $\varepsilon_{t}$ error term. The augmented DickeyFuller test can be performed to check the stationary in the data, and ACF, PACF plots are drawn for stationary time series data.

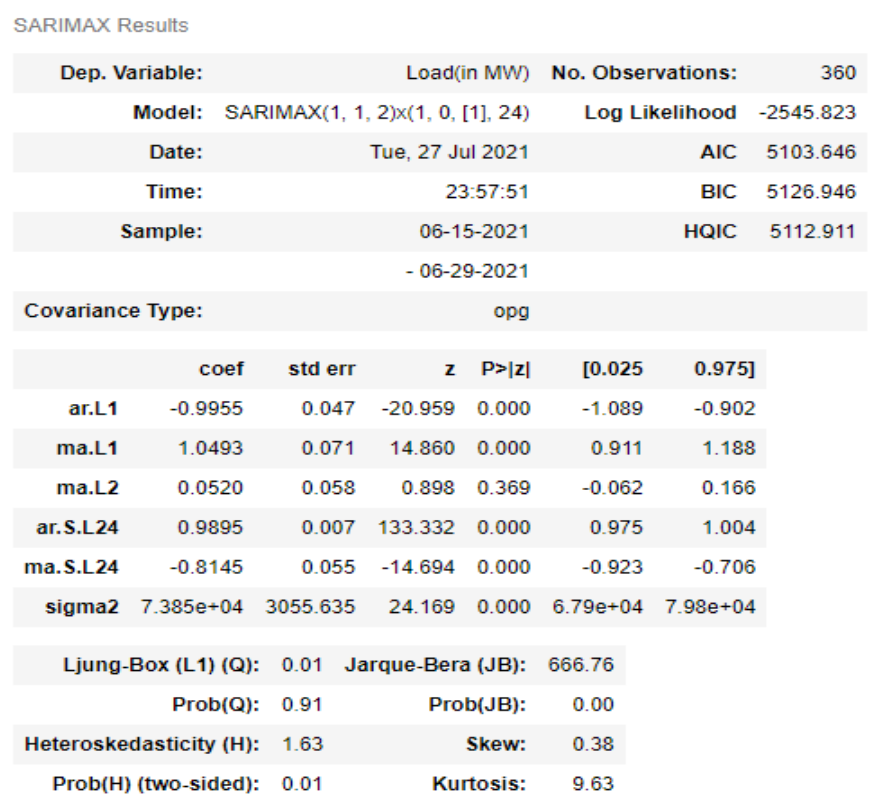

Figure 7: SARIMA Model Results
ACF and PACF plots shown in Figure 8.
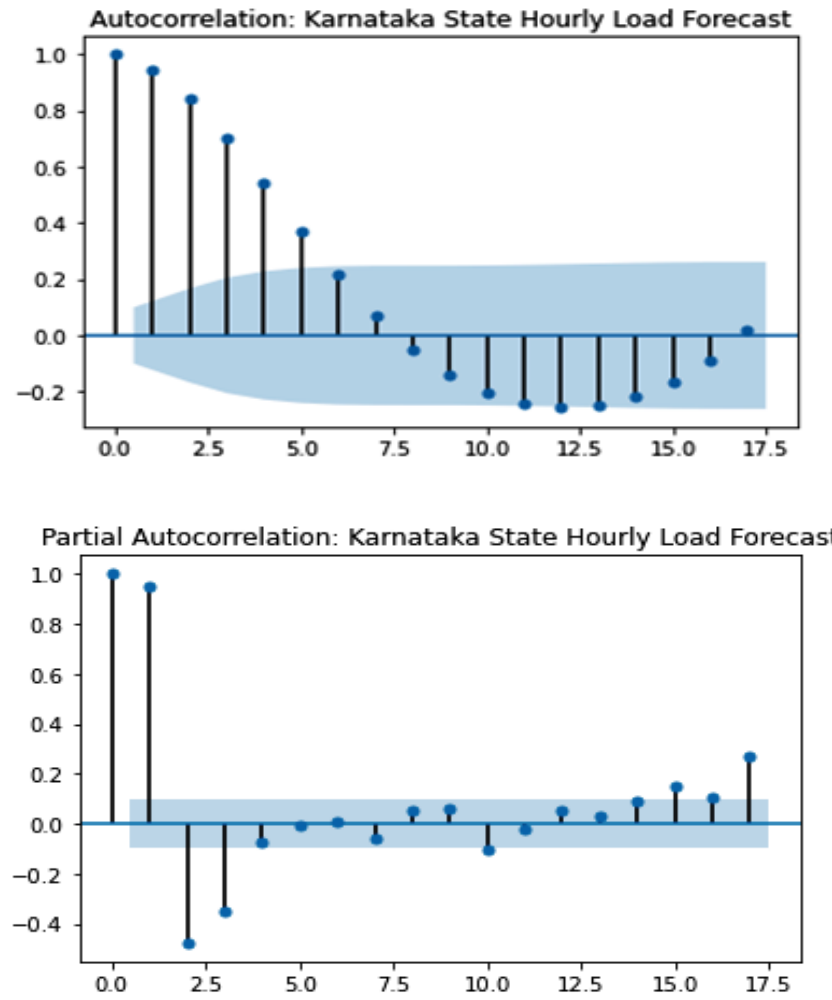

Figure 8: ACF and PACF plots of KS load data set

Figure 9 shows the actual and predicted values of different models.

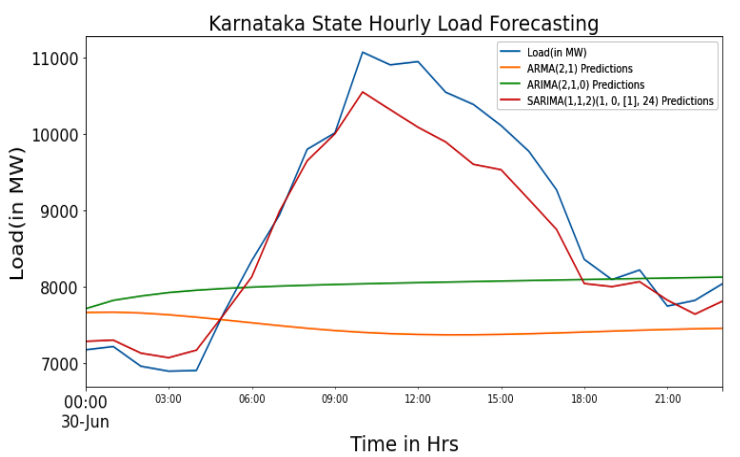

Figure 9: Load forecasting using ARMA, ARIMA, and SARIMA

Figure 10 shows the results of RNN model.

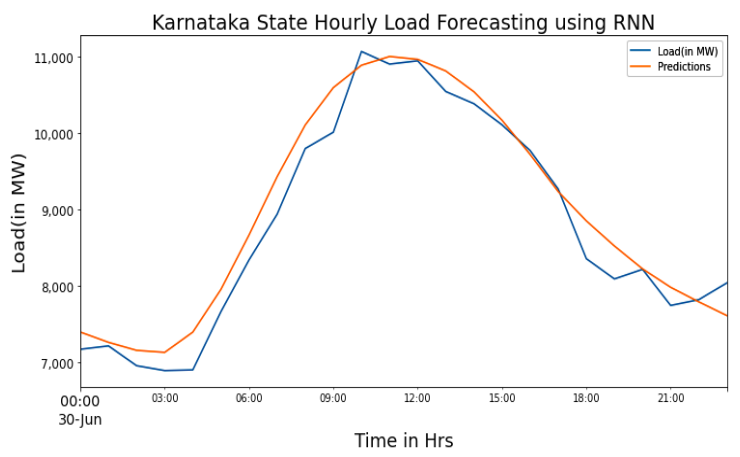

Figure 10: Load forecasting using RNN 
Figure 11 depicts the results using fb Prophet

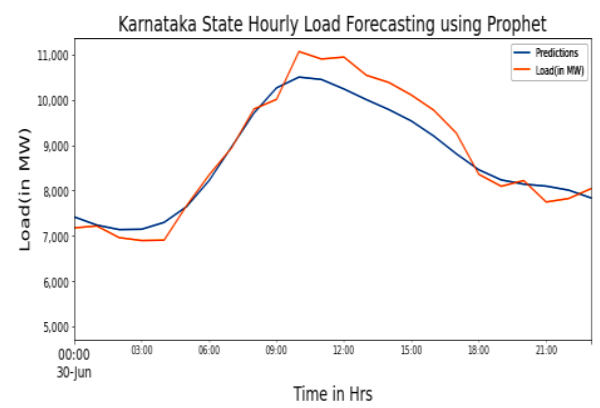

Figure 11: Load forecasting using Prophet

The trend, weekly and daily seasonality of the load using $\mathrm{fb}$ Prophet is given Figure 12.
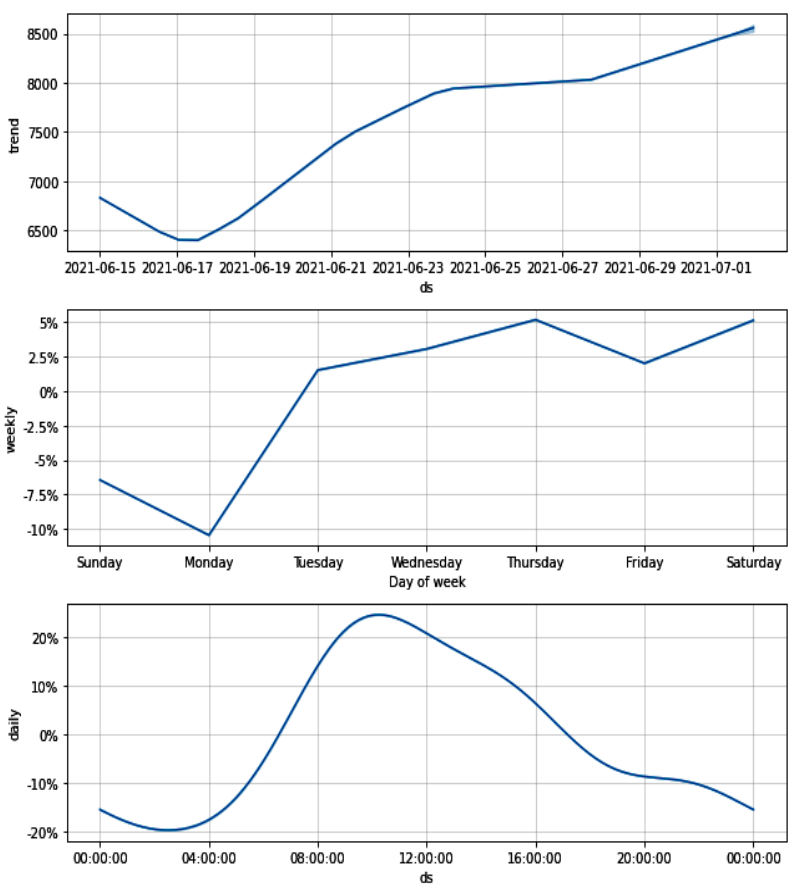

Figure 12: Daily and weekly seasonality

The observed results of different forecasting models are tabulated below,

Table 1: Comparison of Results

\begin{tabular}{|c|c|c|}
\hline Forecast Model & MSE & RMSE (MW) \\
\hline$\overline{\text { ARMA }}$ & 3913970.09 & 1978.37 \\
\hline ARIMA & 2410925.71 & 1552.71 \\
\hline SARIMA & 159894.42 & 399.86 \\
\hline RNN & 86659.53 & 294.37 \\
\hline Prophet & 98125.56 & 313.25 \\
\hline LSTM & 4690.27 & 68.48 \\
\hline
\end{tabular}

\section{CONCLUSION}

In this paper, we developed different techniques to forecast short-term electrical load. Here we have taken KS load consumption dataset, which has 380 data points out of which $90 \%$ of data used for training the network remaining $10 \%$ data used for testing and estimated the load requirement for 24 hours ( 1 day) ahead. From Table 1, the results shows that LSTM networks possess high accuracy and low RMSE values, while ARMA, ARIMA, SARIMA models possess high MSE and RMSE values, and the ability to forecast extreme values is limited and it is difficult to estimate outliers. The problem of vanishing gradient in RNN can be overcome with LSTM.

The mean value of daily load is $7386 \mathrm{MW}$ and the observed RMSE value is $68.48 \mathrm{MW}$, which is as low as the other models. The deviation from the load estimation is only $3.25 \%$. Hence, forecasting electrical load is of the utmost importance to designing a power system to enhance quality of power and reliability.

\section{FUTURE SCOPE}

Further we want to develop AI based energy efficient forecasting model for buildings and gated communities by adopting DSM techniques. The limitation of LSTMs are at the risk of over fitting, and it's difficult to use drop out technique to suppress the issue. This can be solved in our future work.

\section{ACKNOWLEDGMENTS}

Thanks to R\&D centre electrical \& electronics department GND Engineering College, Bidar and VTU-RRC, Belagavi for providing research facilities to carry out the work. Also thanks to KPCL for maintaining the load data sets on web, it's used for the data analysis.

\section{REFERENCES}

[1] M. Ghofrani, M. Ghayekhloo, A. Arabali, and A. Ghayekhloo, "A hybrid short-term load forecasting with a new input selection framework," Energy, vol. 81, pp. 777-786, Mar. 2015.

[2] P. Jiang, F. Liu, and Y. Song, "A hybrid forecasting model based on date-framework strategy and improved feature selection technology for short-term load forecasting," Energy, vol. 119, pp. 694-709, 2017.

[3] Y. Liu, W. Wang, and N. Ghadimi, "Electricity load forecasting by an improved forecast engine for building level consumers," Energy, vol. 139, pp. 18-30, Nov. 2017.

[4] C. Tong, J. Li, C. Lang, F. Kong, J. Niu, and J. J. P. C. Rodrigues, "An efficient deep model for day-ahead electricity load forecasting with stacked denoising auto-encoders," J. Parallel Distrib. Comput., vol. 117, pp. 267-273, Jul. 2018.

[5] G. Hafeez, I. Khan, S. Jan, I. A. Shah, F. A. Khan, and A. Derhab, "A novel hybrid load forecasting framework with intelligent feature engineering and optimization algorithm in smart grid," Appl. Energy, vol. 299 , Oct. 2021

[6] I. Mpawenimana, A. Pegatoquet, V. Roy, L. Rodriguez, and C. Belleudy, "A comparative study of LSTM and ARIMA for energy load prediction with enhanced data preprocessing," in 2020 IEEE Sensors Applications Symposium, SAS 2020 - Proceedings, 2020.

[7] S. Masum, Y. Liu, and J. Chiverton, "Multi-step time series forecasting of electric load using machine learning models," in Lecture Notes in Computer Science (including subseries Lecture Notes in Artificial Intelligence and Lecture Notes in Bioinformatics), 2018, vol. 10841 LNAI. 
[8] X. Liu, Z. Zhang, and Z. Song, "A comparative study of the data-driven day-ahead hourly provincial load forecasting methods: From classical data mining to deep learning," Renew. Sustain. Energy Rev., vol. 119, 2020.

[9] E. Becirovic and M. Cosovic, "Machine learning techniques for shortTerm load forecasting," in 4th International Symposium on Environment Friendly Energies and Applications, EFEA 2016, 2016.

[10] W. He, "Load Forecasting via Deep Neural Networks," in Procedia Computer Science, 2017, vol. 122.

[11] A. O. Hoori, A. Al Kazzaz, R. Khimani, Y. Motai, and A. J. Aved, "Electric Load Forecasting Model Using a Multicolumn Deep Neural Networks," IEEE Trans. Ind. Electron., vol. 67, no. 8, 2020.

[12] S. Hosein and P. Hosein, "Load forecasting using deep neural networks," in 2017 IEEE Power and Energy Society Innovative Smart Grid Technologies Conference, ISGT 2017, 2017.

[13] S. Khan, N. Javaid, A. Chand, R. A. Abbasi, A. B. M. Khan, and H. M. Faisal, "Forecasting day, week and month ahead electricity load consumption of a building using empirical mode decomposition and extreme learning machine," in 2019 15th International Wireless Communications and Mobile Computing Conference, IWCMC 2019, 2019.

[14] H. J. Sadaei, P. C. de Lima e Silva, F. G. Guimarães, and M. H. Lee, "Short-term load forecasting by using a combined method of convolutional neural networks and fuzzy time series," Energy, vol. 175, pp. 365-377, May 2019.

[15] H. Dagdougui, F. Bagheri, H. Le, and L. Dessaint, "Neural network model for short-term and very-short-term load forecasting in district buildings," Energy Build., vol. 203, Nov. 2019.

[16] D. P. Ananthu and K. Neelashetty, "A study of 100k Wp PV Plant Output Power Forecasting: A case study," in Proceedings - 5th International Conference on Computing Methodologies and Communication, ICCMC 2021, 2021.

[17] D. P. Ananthu and K. Neelshetty, "Prominent smart grid measurements by RAP-NN to warrant the reliability of the PV system," Energy Sources, Part A Recover. Util. Environ. Eff., 2019.

[18] S. Siami-Namini, N. Tavakoli, and A. Siami Namin, "A Comparison of ARIMA and LSTM in Forecasting Time Series," in Proceedings - 17th IEEE International Conference on Machine Learning and Applications, ICMLA 2018, 2019, pp. 1394-1401.

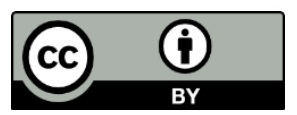

(C) 2021 by the Durga Prasad Ananthu and Prof. Neelashetty K. Submitted for possible open access publication under the terms and conditions of the Creative Commons Attribution (CC BY) license (http://creativecommons.org/licenses/by/4.0/). 